\title{
Effect of Graded Levels of Zinc and Boron on Growth, Yield and Chemical Properties of Soils under Paddy
}

\author{
M.B. Mahendra Kumar ${ }^{1 *}$, C.T. Subbarayappa ${ }^{1}$ and V. Ramamurthy ${ }^{2}$ \\ ${ }^{1}$ Department of Soil Science and Agricultural Chemistry, University of Agricultural Sciences, \\ GKVK, Bengaluru-560065, Karnataka, India \\ ${ }^{2}$ ICAR-NBSS\&LUP, Hebbal Regional Centre, Bengaluru-560024, Karnataka, India \\ *Corresponding author
}

\section{A B S T R A C T}

Keywords

Zinc, Boron, Paddy yield, Soil nutrient status.

\section{Article Info}

Accepted:

10 September 2017

Available Online:

10 October 2017
Field studies were conducted on application of zinc and boron to summer rice crop on Alfisols at Naganahalli village, Mysore district, Karnataka. The results showed that external application of zinc as $\mathrm{ZnSO}_{4} @ 20 \mathrm{~kg} \mathrm{ha}^{-1}$ and boron as borax @ $4 \mathrm{~kg} \mathrm{ha}^{-1}$ significantly enhanced the plant height number of tillers hill ${ }^{-1}$, grains per panicle, thousand grain weight, grain yield and straw yield of paddy. Further there was significant increase in major $(\mathrm{N}, \mathrm{P}$ and $\mathrm{K})$, secondary $(\mathrm{S}, \mathrm{Ca}$ and $\mathrm{Mg}$ ) and micronutrient $(\mathrm{Zn}, \mathrm{Cu}$, $\mathrm{Fe}, \mathrm{Mn}$ and B) status of soils.

\section{Introduction}

Rice is the most important staple food crop of India and particularly Karnataka state. It is grown in India in an area of $43.86 \mathrm{~m}$ ha with a production of $104.80 \mathrm{~m} \mathrm{t}$ with an average productivity of $2.65 \mathrm{t} \mathrm{ha}^{-1}$. In Karnataka it is cultivated in an area of $1.30 \mathrm{~m}$ ha with an annual production of $3.66 \mathrm{~m} \mathrm{t}$ in (Anon., 2015). The yield of rice has stagnated or on the decline as the micronutrients has emerged as yield limiting factors in soils of Karnataka. Further the soils of Karnataka are primarily deficient in zinc and boron and are one of the reasons for stagnation or decline in the yield of rice in southern part of Karnataka. Therefore, efforts are needed to maximize yield of rice by overcoming the deficiencies of these micronutrients (Hafeez et al., 2013).
Now a day's micronutrients deficiency such as zinc and boron is widespread in rice growing areas of country that leads to substantial loss in yield and quality of grains. Soils deficient in micronutrients are not capable of nourishing crop plant successfully and therefore low yield and quality of crops are obtained. Among the micronutrients, zinc and boron play an important role in seed setting and yield of crops. Zinc is required for the biosynthesis of the plant growth regulator such as indole-3-acetic acid (IAA) (Fang et $a l ., 2008$ ) and for carbohydrate and nitrogen metabolism which leads to high yield and yield components. Boron can influence photosynthesis and respiration and activate number of enzymatic systems of protein and 
nucleic acid metabolism in plants (Chowdhury et al., 2010).For proper growth and development of crop plants, zinc and boron are essential micronutrients to improve the economic yield and quality of several crop plants (Pratima Sinha et al., 2000).

Fertilizers particularly zinc and boron in addition to recommended dose of major nutrients is needed to increase yield, uptake and total content of essential nutrients in rice (Abbas et al., 2013). Therefore, the experiment was undertaken with an objective to find out the effect of graded levels of zinc and boron on growth, yield and chemical properties of rice soils.

\section{Materials and Methods}

Field experiment was conducted on farmer's field at Naganahalli village, Mysore district, Karnataka during summer season, to study the effect of graded levels of zinc and boron on growth, yield and chemical properties of soils under paddy. A composite surface soil sample (0-15 cm depth) was collected for analysis before the commencement of the experiment.

The initial soil status of the experimental site is presented in Table 1. The experiment was laid out in randomised complete block design (RCBD) with sixteen treatments (16) and replicated thrice (3) with using Jaya variety of paddy.

Zinc and boron were added through $\mathrm{ZnSO}_{4}$ and borax respectively at three levels viz., 10 , 20 and $30 \mathrm{~kg} \mathrm{ZnSO}_{4} \mathrm{ha}^{-1}$ and 2, 4 and $6 \mathrm{~kg}$ borax $\mathrm{ha}^{-1}$. At transplanting, recommended dose of FYM, 50 per cent of the nitrogen, 100 percent of phosphorus and potassium were applied as complex 20:20:20 fertilizer. The remaining nitrogen was given in two equal split doses through urea, 25 per cent each at tillering and panicle initiation stages, respectively.
Twenty three days old rice seedlings were transplanted at a spacing of $20 \times 10 \mathrm{~cm}$ with 2 to 3 seedlings per hill in well puddled and leveled plots. Plots were irrigated after two days of transplanting to maintain $2.0 \mathrm{~cm}$ level of submergence for eight days. Later, these plots were irrigated to maintain the water level to a height of $5 \mathrm{~cm}$ throughout the crop growth except last ten days to harvest.

Appropriate plant protection measures were taken during crop growth. Soil samples (0-15 $\mathrm{cm}$ depth) from individual plots were collected at panicle initiation stage and at harvest for analysis of physico-chemical properties of soil by standard method (Piper, 1966; Jackson, 1973; Walkley and Black, 1934; Subbaiah and Asija, 1956; Page et al., 1982; Lindsay and Norwell, 1978 and Berger and Troug, 1939). The biometric observations like plant height at panicle initiation stage and yield parameters viz., number of tillers, number of grains panicle ${ }^{-1}$, test weight $(\mathrm{g})$, grain and straw yield were recorded plot wise. Post-harvest soil samples were collected from different treatments and analysed for nutrient status.

\section{Results and Discussion}

\section{Growth and yield parameters of paddy}

The results presented in Table 2 indicate that application of recommended levels of major nutrient fertilizers (NPK) along with $\mathrm{ZnSO}_{4}$ @ $20 \mathrm{~kg} \mathrm{ha}^{-1}$ and Borax @ $4 \mathrm{~kg} \mathrm{ha}^{-1}$ has significantly enhanced the plant height (87.21 $\mathrm{cm})$ and was superior over all other treatments. Lower plant height of $64.67 \mathrm{~cm}$ was recorded in $\mathrm{T}_{1}$ treatment $(\mathrm{RDF}+\mathrm{FYM})$. It is a well-known fact that boron is essential in enhancing carbohydrate metabolism, sugar transport, cell wall structure, protein metabolism, root growth and stimulating other physiological processes of plant (Ashour and Reda, 1972). Since the soil was 
deficient in both DTPA Zn and B the paddy crop responded well and resulted in enhanced growth and yield. These results were in accordance with that of Balachandar et al., (2003) and Jyoti Sharma et al., (2013) who reported that increase in plant height of crop due to the application of boron and zinc. In this study, a synergistic effect of boron and zinc was found in increasing plant height of rice.

Significantly highest number of tillers hill ${ }^{-1}$ (26.88 number of tillers hill $^{-1}$ ), number of grains per panicle (130.05 grains per panicle) and thousand grain weight (26.96 g) were recorded in $\mathrm{T}_{12}$ treatment which received RDF + $\mathrm{ZnSO}_{4} @ 20 \mathrm{~kg} \mathrm{ha}^{-1}+$ Borax @ $4 \mathrm{~kg} \mathrm{ha}^{-1}$ which was superior over all other treatments. The lowest number of tillers hill $^{-1}$ (12.61 number of tillers hill ${ }^{-1}$ ), number of grains per panicle (100.42 grains per panicle) and thousand grain weight $(21.87 \mathrm{~g})$ was recorded in $\mathrm{T}_{1}$ treatment which received $\mathrm{RDF}+\mathrm{FYM}$ as compared to other treatments. The results very clearly indicated that the application of both the nutrients may increases the number of tillers per hill and also the need for application of boron at an early stage of the crop to increase the tiller numbers. These results were in accordance with that of Muhammad et al., (2012).

A significant increase in grain and straw yield of rice was noticed over the $\mathrm{RDF}+\mathrm{FYM}$ due to application of different levels of $\mathrm{ZnSO}_{4}$ and borax. The highest grain (62.21 q ha $\left.\mathrm{q}^{-1}\right)$ and straw (88.33 $\mathrm{q} \mathrm{ha}^{-1}$ ) yield was recorded in the $\mathrm{T}_{12}$ treatment which received $\mathrm{RDF}+\mathrm{ZnSO}_{4}$ @ $20 \mathrm{~kg} \mathrm{ha}^{-1}+$ Borax @ $4 \mathrm{~kg} \mathrm{ha}^{-1}$ which was significantly superior over all other treatments. The lowest grain (49.89 $\left.\mathrm{q} \mathrm{ha}^{-1}\right)$ and straw yield (64.40 $\left.\mathrm{q} \mathrm{ha}^{-1}\right)$ was recorded in $\mathrm{T}_{1}(\mathrm{RDF}+\mathrm{FYM})$ treatment. Application of $\mathrm{Zn}$ and $\mathrm{B}$, when used alone as well as when applied in combination, resulted in significantly higher grain and straw yields than the control. The beneficial effect of B on enhancement of crop yield has been reported by Sharma (1995), Christos Dordas (2006) and Raghuveer Rao et al., (2013). Similarly, the favorable effect of $\mathrm{Zn}$ on yield of different crops has also been well documented (Das, 1992, Subrahmaniyan et al., 2001 and Bagewadi et al., 2003). In this experiment, the crop yield increased to a much greater extent due to the combined use of $\mathrm{Zn}$ and $\mathrm{B}$ than their use alone. These results were in accordance with that of Muhammad et al., (2012), Quddus et al., (2011) and Jyoti Sharma et al., (2013).

\section{Chemical properties of soil}

\section{Soil pH, EC and OC}

The results pertaining to soil chemical properties are presented in Table 3. There was no significant difference in soil $\mathrm{pH}, \mathrm{EC}$ and OC content in soil at both stages of crop growth.

\section{Available major and secondary nutrient status of soil at panicle initiation and at harvest stages of paddy}

The status of major and secondary nutrient in soil as influenced by graded levels of zinc and boron in paddy are presented in Table 4 and Table 5. Increase in available nitrogen was noticed among treatments in both stages due to application of RDF with zinc and boron.

A significant increase in available nitrogen was noticed over RDF+FYM treatment due to combined application of NPK with $\mathrm{Zn}$ and B which form synergistic relationship and helps in increased available nitrogen in all treated plots. The available nitrogen in soil was higher at panicle initiation stage of crop and declined at later stage. This might be due to the uptake of $\mathrm{N}$ by the growing plants as described by Prakash et al., (1994). 
There was a significant increase in available phosphorus status of soil over RDF with borax treated plots in both stages. The boron had no relation with $\mathrm{P}$ compared to $\mathrm{Zn}$.

But the combination of RDF with $\mathrm{ZnSO}_{4}$ and Borax had slight increase in available $\mathrm{P}$ compared to initial value might be due to addition of $\mathrm{P}$ fertilizer and $\mathrm{S}$ in $\mathrm{ZnSO}_{4}$ that helps in $\mathrm{P}$ availability.

The available $\mathrm{P}$ content was higher in initial stages of crop but declined at later stages. This may be due to uptake of $\mathrm{P}$ by growing plants and/or due to refixation of solubilized P.
These observations are in conformity with the findings of Dashrath Singh et al., (1976).

Available potassium showed significant difference among treatments. Significantly higher potassium content was observed in $\mathrm{T}_{16}$ treatment $\left(\mathrm{RDF}+\mathrm{ZnSO}_{4} @ 30 \mathrm{~kg} \mathrm{ha}^{-1}+\right.$ Borax@6 kg ha ${ }^{-1}$ ) at both stages and followed by $\mathrm{T}_{13}$ treatment $\left(\mathrm{RDF}+\mathrm{ZnSO}_{4} @\right.$ $20 \mathrm{~kg} \mathrm{ha}^{-1}+$ Borax @6 kg ha $\left.{ }^{-1}\right)$ at both stages. $\mathrm{RDF}+\mathrm{FYM}\left(\mathrm{T}_{1}\right)$ recorded significantly lower available potassium at both stages. Rao and Shukla (1997) reported that increase in release rate of $\mathrm{K}$ on application of fertilizers resulted in larger decline of $\mathrm{K}$ in reserve pool of the soil.

Table.1 Initial physical and chemical properties of the experimental site

\begin{tabular}{|c|c|c|}
\hline \multicolumn{2}{|l|}{ Particulars } & Content \\
\hline \multicolumn{3}{|c|}{ Physical properties } \\
\hline \multirow{4}{*}{$\begin{array}{l}\text { Particle size } \\
\text { distribution }\end{array}$} & Sand (\%) & 78.00 \\
\hline & Silt (\%) & 7.50 \\
\hline & Clay (\%) & 14.50 \\
\hline & Texture & Sandy loam \\
\hline \multicolumn{2}{|c|}{ Bulk density $\left(\mathrm{Mg} \mathrm{m}^{-3}\right)$} & 1.53 \\
\hline \multicolumn{2}{|c|}{ Particle density $\left(\mathrm{Mg} \mathrm{m}^{-3}\right)$} & 2.70 \\
\hline \multicolumn{2}{|c|}{ Pore space $(\%)$} & 44.00 \\
\hline \multirow{2}{*}{\multicolumn{3}{|c|}{$\begin{array}{l}\text { Maximum water holding capacity (\%) } \\
\text { Chemical properties }\end{array}$}} \\
\hline & & \\
\hline \multicolumn{2}{|c|}{$\mathrm{pH}(1: 2.5)$} & 7.38 \\
\hline \multicolumn{2}{|c|}{$\mathrm{EC}\left(\mathrm{dSm}^{-1}\right.$ at $\left.25^{0} \mathrm{C}\right)$} & 0.29 \\
\hline \multicolumn{2}{|c|}{ Organic Carbon $(\%)$} & 0.51 \\
\hline \multicolumn{2}{|c|}{ Availabe $\mathrm{N}\left(\mathrm{kg} \mathrm{ha}^{-1}\right)$} & 287.28 \\
\hline \multicolumn{2}{|c|}{ Available $\mathrm{P}_{2} \mathrm{O}_{5}\left(\mathrm{~kg} \mathrm{ha}^{-1}\right)$} & 17.58 \\
\hline \multicolumn{2}{|c|}{ Available $\mathrm{K}_{2} \mathrm{O}\left(\mathrm{kg} \mathrm{ha}^{-1}\right)$} & 206.04 \\
\hline \multicolumn{2}{|c|}{$\mathrm{CEC}\left[\mathrm{Cmol}\left(\mathrm{P}^{+}\right) \mathrm{kg}^{-1}\right]$} & 14.80 \\
\hline \multicolumn{2}{|c|}{ Exchangeable $\mathrm{Ca}\left[\mathrm{Cmol}\left(\mathrm{P}^{+}\right) \mathrm{kg}^{-1}\right]$} & 5.86 \\
\hline \multicolumn{2}{|c|}{ Exchangeable $\mathrm{Mg}\left[\mathrm{Cmol}\left(\mathrm{P}^{+}\right) \mathrm{kg}^{-1}\right]$} & 2.50 \\
\hline \multicolumn{2}{|c|}{ Available $\mathrm{S}\left(\mathrm{mg} \mathrm{kg}^{-1}\right)$} & 14.40 \\
\hline \multicolumn{2}{|c|}{ DTPA - Extractable $\mathrm{Zn}\left(\mathrm{mg} \mathrm{kg}^{-1}\right)$} & 0.92 \\
\hline \multicolumn{2}{|c|}{ DTPA - Extractable $\mathrm{Cu}\left(\mathrm{mg} \mathrm{kg}^{-1}\right)$} & 1.20 \\
\hline \multicolumn{2}{|c|}{ DTPA - Extractable Fe $\left(\mathrm{mg} \mathrm{kg}^{-1}\right)$} & 3.24 \\
\hline \multicolumn{2}{|c|}{ DTPA - Extractable $\mathrm{Mn}\left(\mathrm{mg} \mathrm{kg}^{-1}\right)$} & 2.01 \\
\hline \multicolumn{2}{|c|}{ Hot water soluble $\mathrm{B}\left(\mathrm{mg} \mathrm{kg}^{-1}\right)$} & 0.69 \\
\hline
\end{tabular}


Table.2 Effect of graded levels of zinc and boron on growth and yield ( $\mathrm{q} \mathrm{ha}^{-1}$ ) of paddy at Naganahalli, Mysore district, Karnataka

\begin{tabular}{|c|c|c|c|c|c|c|}
\hline Treatments & $\begin{array}{l}\text { Plant height } \\
\text { (cm) }\end{array}$ & $\begin{array}{c}\text { Number } \\
\text { of tillers } \\
\text { hill }^{-1}\end{array}$ & $\begin{array}{l}\text { No.of grains } \\
\text { / panicle }\end{array}$ & $\begin{array}{l}\text { Thousand } \\
\text { grain } \\
\text { weight }(\mathrm{g})\end{array}$ & $\begin{array}{l}\text { Grain yield } \\
\left.(\mathrm{q} \mathrm{ha})^{-1}\right)\end{array}$ & $\begin{array}{c}\text { Straw } \\
\text { yield } \\
\left(\mathrm{q} \mathrm{ha}^{-1)}\right.\end{array}$ \\
\hline $\mathrm{T}_{1}:$ Rec. NPK + FYM (No Zn and B application) & 64.67 & 12.61 & 100.42 & 21.87 & 49.89 & 64.40 \\
\hline $\mathrm{T}_{2}: \mathrm{RDF}+\mathrm{ZnSO}_{4} @ 10 \mathrm{~kg} \mathrm{ha}^{-1}$ & 73.11 & 17.65 & 108.03 & 23.02 & 50.74 & 70.16 \\
\hline $\mathrm{T}_{3}: \mathrm{RDF}+\mathrm{ZnSO}_{4} @ 20 \mathrm{~kg} \mathrm{ha}^{-1}$ & 78.47 & 20.66 & 119.36 & 24.68 & 52.25 & 74.99 \\
\hline $\mathrm{T}_{4}: \mathrm{RDF}+\mathrm{ZnSO}_{4} @ 30 \mathrm{~kg} \mathrm{ha}^{-1}$ & 75.40 & 19.87 & 117.67 & 23.40 & 51.15 & 71.13 \\
\hline $\mathrm{T}_{5}: \mathrm{RDF}+$ Borax @ $2 \mathrm{~kg} \mathrm{ha}^{-1}$ & 73.31 & 17.21 & 112.00 & 23.20 & 52.39 & 75.64 \\
\hline $\mathrm{T}_{6}: \mathrm{RDF}+$ Borax @ $4 \mathrm{~kg} \mathrm{ha}^{-1}$ & 77.67 & 20.93 & 120.33 & 25.14 & 54.25 & 80.08 \\
\hline $\mathrm{T}_{7}: \mathrm{RDF}+$ Borax @6 kg ha ${ }^{-1}$ & 75.65 & 20.22 & 119.84 & 24.16 & 53.42 & 79.46 \\
\hline $\begin{array}{l}\mathrm{T}_{8}: \mathrm{RDF}+\mathrm{ZnSO}_{4} @ 10 \mathrm{~kg} \mathrm{ha}^{-1}+\text { Borax @2 kg } \\
\mathrm{ha}^{-1}\end{array}$ & 78.10 & 21.44 & 120.86 & 23.57 & 54.58 & 77.38 \\
\hline $\begin{array}{l}\mathrm{T}_{9}: \mathrm{RDF}+\mathrm{ZnSO}_{4} @ 10 \mathrm{~kg} \mathrm{ha}^{-1}+\text { Borax @ } 4 \mathrm{~kg} \\
\mathrm{ha}^{-1}\end{array}$ & 80.44 & 23.67 & 121.77 & 25.20 & 55.14 & 81.10 \\
\hline $\begin{array}{l}\mathrm{T}_{10}: \mathrm{RDF}+\mathrm{ZnSO}_{4} @ 10 \mathrm{~kg} \mathrm{ha}^{-1}+\text { Borax @ } 6 \mathrm{~kg} \\
\mathrm{ha}^{-1}\end{array}$ & 81.85 & 23.45 & 122.20 & 24.71 & 56.70 & 84.53 \\
\hline $\begin{array}{l}\mathrm{T}_{11}: \mathrm{RDF}+\mathrm{ZnSO}_{4} @ 20 \mathrm{~kg} \mathrm{ha}^{-1}+\text { Borax @ } 2 \mathrm{~kg} \\
\mathrm{ha}^{-1}\end{array}$ & 83.18 & 22.32 & 127.59 & 25.32 & 60.94 & 86.25 \\
\hline $\begin{array}{l}\text { T12: RDF+ } \mathrm{ZnSO}_{4} @ 20 \mathrm{~kg} \mathrm{ha}^{-1}+\text { Borax @ } 4 \mathrm{~kg} \\
\mathrm{ha}^{-1}\end{array}$ & 87.21 & 26.88 & 130.05 & 26.96 & 62.21 & 88.33 \\
\hline $\begin{array}{l}\mathrm{T}_{13}: \mathrm{RDF}+\mathrm{ZnSO}_{4} @ 20 \mathrm{~kg} \mathrm{ha}^{-1}+\text { Borax @6 kg } \\
\mathrm{ha}^{-1}\end{array}$ & 85.87 & 23.85 & 129.14 & 26.33 & 61.63 & 87.43 \\
\hline $\begin{array}{l}\mathrm{T}_{14}: \mathrm{RDF}+\mathrm{ZnSO}_{4} @ 30 \mathrm{~kg} \mathrm{ha}^{-1}+\text { Borax @ } 2 \mathrm{~kg} \\
\mathrm{ha}^{-1}\end{array}$ & 84.57 & 23.46 & 123.50 & 24.98 & 60.35 & 84.27 \\
\hline $\begin{array}{l}\mathrm{T}_{15}: \mathrm{RDF}+\mathrm{ZnSO}_{4} @ 30 \mathrm{~kg} \mathrm{ha}^{-1}+\text { Borax @ } 4 \mathrm{~kg} \\
\mathrm{ha}^{-1}\end{array}$ & 81.11 & 22.46 & 122.67 & 24.52 & 59.36 & 83.82 \\
\hline $\begin{array}{l}\mathrm{T}_{16}: \mathrm{RDF}+\mathrm{ZnSO}_{4} @ 30 \mathrm{~kg} \mathrm{ha}^{-1}+\text { Borax @ } 6 \mathrm{~kg} \\
\mathrm{ha}^{-1}\end{array}$ & 80.66 & 21.58 & 122.01 & 23.25 & 58.75 & 81.61 \\
\hline S.Em \pm & 0.88 & 0.51 & 1.67 & 0.37 & 1.18 & 0.35 \\
\hline C.D. at $5 \%$ & 2.64 & 1.53 & 5.00 & 1.12 & 3.55 & 1.05 \\
\hline
\end{tabular}


Table.3 Effect of graded levels of zinc and boron on soil reaction $(\mathrm{pH})$, electrical conductivity (EC) and organic carbon content of soil at different growth stages of paddy at Naganahalli, Mysore district, Karnataka

\begin{tabular}{|c|c|c|c|c|c|c|}
\hline \multirow[t]{2}{*}{ Treatments } & \multicolumn{2}{|c|}{$\mathrm{pH}$} & \multicolumn{2}{|c|}{$\mathrm{EC}\left(\mathrm{dSm}^{-1}\right)$} & \multicolumn{2}{|c|}{$\mathrm{OC}(\%)$} \\
\hline & $\begin{array}{l}\text { Panicle } \\
\text { initiation } \\
\text { stage }\end{array}$ & $\begin{array}{c}\text { Harvest } \\
\text { stage }\end{array}$ & $\begin{array}{l}\text { Panicle } \\
\text { initiation } \\
\text { stage }\end{array}$ & $\begin{array}{c}\text { Harvest } \\
\text { stage }\end{array}$ & $\begin{array}{l}\text { Panicle } \\
\text { initiation } \\
\text { stage }\end{array}$ & $\begin{array}{l}\text { Harvest } \\
\text { stage }\end{array}$ \\
\hline $\mathrm{T}_{1}:$ Rec. NPK + FYM (No Zn and B application) & 7.37 & 7.34 & 0.31 & 0.29 & 0.66 & 0.61 \\
\hline $\mathrm{T}_{2}: \mathrm{RDF}+\mathrm{ZnSO}_{4} @ 10 \mathrm{~kg} \mathrm{ha}^{-1}$ & 7.19 & 7.20 & 0.33 & 0.30 & 0.63 & 0.58 \\
\hline $\mathrm{T}_{3}: \mathrm{RDF}+\mathrm{ZnSO}_{4} @ 20 \mathrm{~kg} \mathrm{ha}^{-1}$ & 7.17 & 7.18 & 0.34 & 0.32 & 0.60 & 0.55 \\
\hline $\mathrm{T}_{4}: \mathrm{RDF}+\mathrm{ZnSO}_{4} @ 30 \mathrm{~kg} \mathrm{ha}^{-1}$ & 7.10 & 7.12 & 0.35 & 0.33 & 0.59 & 0.52 \\
\hline $\mathrm{T}_{5}: \mathrm{RDF}+$ Borax @ $2 \mathrm{~kg} \mathrm{ha}^{-1}$ & 7.25 & 7.22 & 0.30 & 0.29 & 0.57 & 0.54 \\
\hline $\mathrm{T}_{6}: \mathrm{RDF}+$ Borax @ 4 kg ha ${ }^{-1}$ & 7.22 & 7.20 & 0.32 & 0.31 & 0.62 & 0.57 \\
\hline $\mathrm{T}_{7}: \mathrm{RDF}+$ Borax @ $6 \mathrm{~kg} \mathrm{ha}^{-1}$ & 7.15 & 7.13 & 0.33 & 0.32 & 0.59 & 0.55 \\
\hline $\mathrm{T}_{8}: \mathrm{RDF}+\mathrm{ZnSO}_{4} @ 10 \mathrm{~kg} \mathrm{ha}^{-1}+$ Borax @ $2 \mathrm{~kg} \mathrm{ha}^{-1}$ & 7.26 & 7.23 & 0.34 & 0.33 & 0.58 & 0.54 \\
\hline $\mathrm{T}_{9}: \mathrm{RDF}+\mathrm{ZnSO}_{4} @ 10 \mathrm{~kg} \mathrm{ha}^{-1}+$ Borax @ $4 \mathrm{~kg} \mathrm{ha}^{-1}$ & 7.24 & 7.20 & 0.35 & 0.34 & 0.57 & 0.53 \\
\hline $\mathrm{T}_{10}: \mathrm{RDF}+\mathrm{ZnSO}_{4} @ 10 \mathrm{~kg} \mathrm{ha}^{-1}+$ Borax @6 kg ha ${ }^{-1}$ & 7.20 & 7.17 & 0.36 & 0.33 & 0.54 & 0.51 \\
\hline $\mathrm{T}_{11}: \mathrm{RDF}+\mathrm{ZnSO}_{4} @ 20 \mathrm{~kg} \mathrm{ha}^{-1}+$ Borax @2 $\mathrm{kg} \mathrm{ha}^{-1}$ & 7.24 & 7.21 & 0.34 & 0.32 & 0.57 & 0.55 \\
\hline $\mathrm{T}_{12}: \mathrm{RDF}+\mathrm{ZnSO}_{4} @ 20 \mathrm{~kg} \mathrm{ha}^{-1}+$ Borax @ $4 \mathrm{~kg} \mathrm{ha}^{-1}$ & 7.21 & 7.18 & 0.37 & 0.35 & 0.55 & 0.52 \\
\hline $\mathrm{T}_{13}: \mathrm{RDF}+\mathrm{ZnSO}_{4} @ 20 \mathrm{~kg} \mathrm{ha}^{-1}+$ Borax @6 kg ha ${ }^{-1}$ & 7.19 & 7.15 & 0.38 & 0.36 & 0.55 & 0.51 \\
\hline $\mathrm{T}_{14}: \mathrm{RDF}+\mathrm{ZnSO}_{4} @ 30 \mathrm{~kg} \mathrm{ha}^{-1}+$ Borax @ $2 \mathrm{~kg} \mathrm{ha}^{-1}$ & 7.18 & 7.16 & 0.35 & 0.33 & 0.56 & 0.53 \\
\hline $\mathrm{T}_{15}: \mathrm{RDF}+\mathrm{ZnSO}_{4} @ 30 \mathrm{~kg} \mathrm{ha}^{-1}+$ Borax @ $4 \mathrm{~kg} \mathrm{ha}^{-1}$ & 7.14 & 7.13 & 0.37 & 0.35 & 0.53 & 0.51 \\
\hline $\mathrm{T}_{16}: \mathrm{RDF}+\mathrm{ZnSO}_{4} @ 30 \mathrm{~kg} \mathrm{ha}^{-1}+$ Borax @ $6 \mathrm{~kg} \mathrm{ha}^{-1}$ & 7.08 & 7.04 & 0.39 & 0.36 & 0.52 & 0.50 \\
\hline S.Em \pm & 0.05 & 0.03 & 0.01 & 0.01 & 0.02 & 0.02 \\
\hline C.D. at $5 \%$ & NS & 0.10 & 0.04 & 0.03 & 0.06 & 0.05 \\
\hline
\end{tabular}


Table.4 Effect of graded levels of zinc and boron on available major nutrient status $\left(\mathrm{kg} \mathrm{ha}^{-1}\right)$ of soil at different growth stages of paddy at Naganahalli, Mysore district, Karnataka

\begin{tabular}{|c|c|c|c|c|c|c|}
\hline \multirow[t]{2}{*}{ Treatments } & \multicolumn{2}{|c|}{ Available N } & \multicolumn{2}{|c|}{ Available $\mathrm{P}_{2} \mathrm{O}_{5}$} & \multicolumn{2}{|c|}{ Available $\mathrm{K}_{2} \mathrm{O}$} \\
\hline & $\begin{array}{c}\text { Panicle } \\
\text { initiation } \\
\text { stage }\end{array}$ & $\begin{array}{c}\text { Harvest } \\
\text { stage }\end{array}$ & $\begin{array}{c}\text { Panicle } \\
\text { initiation } \\
\text { stage }\end{array}$ & $\begin{array}{c}\text { Harvest } \\
\text { stage }\end{array}$ & $\begin{array}{c}\text { Panicle } \\
\text { initiation } \\
\text { stage }\end{array}$ & $\begin{array}{c}\text { Harvest } \\
\text { stage }\end{array}$ \\
\hline $\mathrm{T}_{1}:$ Rec. NPK + FYM (No Zn and B application) & 289.93 & 280.50 & 17.82 & 17.70 & 210.38 & 206.72 \\
\hline $\mathrm{T}_{2}: \mathrm{RDF}+\mathrm{ZnSO}_{4} @ 10 \mathrm{~kg} \mathrm{ha}^{-1}$ & 297.53 & 287.83 & 18.78 & 18.39 & 212.66 & 207.00 \\
\hline $\mathrm{T}_{3}: \mathrm{RDF}+\mathrm{ZnSO}_{4} @ 20 \mathrm{~kg} \mathrm{ha}^{-1}$ & 305.21 & 296.18 & 18.47 & 18.08 & 214.95 & 210.27 \\
\hline $\mathrm{T}_{4}: \mathrm{RDF}+\mathrm{ZnSO}_{4} @ 30 \mathrm{~kg} \mathrm{ha}^{-1}$ & 299.80 & 291.65 & 18.03 & 17.82 & 217.46 & 212.63 \\
\hline $\mathrm{T}_{5}: \mathrm{RDF}+$ Borax @ $2 \mathrm{~kg} \mathrm{ha}^{-1}$ & 297.64 & 287.55 & 16.08 & 15.73 & 218.33 & 214.27 \\
\hline $\mathrm{T}_{6}: \mathrm{RDF}+$ Borax @ $4 \mathrm{~kg} \mathrm{ha}^{-1}$ & 301.78 & 293.68 & 16.71 & 16.11 & 220.48 & 217.30 \\
\hline $\mathrm{T}_{7}: \mathrm{RDF}+$ Borax @6 kg ha ${ }^{-1}$ & 303.05 & 294.64 & 17.03 & 16.56 & 219.06 & 214.37 \\
\hline $\mathrm{T}_{8}: \mathrm{RDF}+\mathrm{ZnSO}_{4} @ 10 \mathrm{~kg} \mathrm{ha}^{-1}+$ Borax @2 kg ha ${ }^{-1}$ & 309.38 & 298.53 & 18.63 & 17.92 & 219.42 & 214.92 \\
\hline $\mathrm{T}_{9}: \mathrm{RDF}+\mathrm{ZnSO}_{4} @ 10 \mathrm{~kg} \mathrm{ha}^{-1}+$ Borax @ $4 \mathrm{~kg} \mathrm{ha}^{-1}$ & 312.19 & 304.77 & 19.35 & 18.44 & 222.03 & 216.31 \\
\hline $\mathrm{T}_{10}: \mathrm{RDF}+\mathrm{ZnSO}_{4} @ 10 \mathrm{~kg} \mathrm{ha}^{-1}+$ Borax @ $6 \mathrm{~kg} \mathrm{ha}^{-1}$ & 316.98 & 307.47 & 19.82 & 18.77 & 223.28 & 216.28 \\
\hline $\mathrm{T}_{11}: \mathrm{RDF}+\mathrm{ZnSO}_{4} @ 20 \mathrm{~kg} \mathrm{ha}^{-1}+$ Borax @2 kg ha ${ }^{-1}$ & 317.78 & 304.90 & 21.39 & 19.36 & 220.37 & 212.24 \\
\hline $\mathrm{T}_{12}: \mathrm{RDF}+\mathrm{ZnSO}_{4} @ 20 \mathrm{~kg} \mathrm{ha}^{-1}+$ Borax @ $4 \mathrm{~kg} \mathrm{ha}^{-1}$ & 322.89 & 312.03 & 22.12 & 20.89 & 222.48 & 217.13 \\
\hline $\mathrm{T}_{13}: \mathrm{RDF}+\mathrm{ZnSO}_{4} @ 20 \mathrm{~kg} \mathrm{ha}^{-1}+$ Borax @ $6 \mathrm{~kg} \mathrm{ha}^{-1}$ & 322.89 & 309.50 & 22.04 & 19.72 & 224.57 & 217.78 \\
\hline $\mathrm{T}_{14}: \mathrm{RDF}+\mathrm{ZnSO}_{4} @ 30 \mathrm{~kg} \mathrm{ha}^{-1}+$ Borax @2 kg ha ${ }^{-1}$ & 322.18 & 311.88 & 20.93 & 19.39 & 220.60 & 212.60 \\
\hline $\mathrm{T}_{15}: \mathrm{RDF}+\mathrm{ZnSO}_{4} @ 30 \mathrm{~kg} \mathrm{ha}^{-1}+$ Borax @ $4 \mathrm{~kg} \mathrm{ha}^{-1}$ & 318.49 & 311.27 & 20.89 & 19.00 & 223.09 & 216.71 \\
\hline $\mathrm{T}_{16}: \mathrm{RDF}+\mathrm{ZnSO}_{4} @ 30 \mathrm{~kg} \mathrm{ha}^{-1}+$ Borax @ $6 \mathrm{~kg} \mathrm{ha}^{-1}$ & 313.57 & 308.33 & 20.26 & 18.98 & 225.66 & 218.28 \\
\hline S.Em \pm & 2.58 & 1.21 & 0.32 & 0.18 & 1.47 & 1.46 \\
\hline C.D. at $5 \%$ & 7.74 & 3.64 & 0.97 & 0.53 & 4.39 & 4.36 \\
\hline
\end{tabular}


Table.5 Effect of graded levels of zinc and boron on secondary nutrient status of soil at different growth stages of paddy at Naganahalli, Mysore district, Karnataka

\begin{tabular}{|c|c|c|c|c|c|c|}
\hline \multirow[b]{2}{*}{ Treatments } & \multicolumn{2}{|c|}{$\mathrm{Ca}\left[\mathrm{Cmol}\left(\mathrm{P}^{+}\right) \mathrm{kg}^{-1}\right]$} & \multicolumn{2}{|c|}{$\operatorname{Mg}\left[\mathrm{Cmol}\left(\mathrm{P}^{+}\right) \mathrm{kg}^{-1}\right]$} & \multicolumn{2}{|c|}{ Available $\mathrm{S}\left(\mathrm{mg} \mathrm{kg}^{-1}\right)$} \\
\hline & $\begin{array}{l}\text { Panicle } \\
\text { initiation } \\
\text { stage }\end{array}$ & $\begin{array}{c}\text { Harvest } \\
\text { stage }\end{array}$ & $\begin{array}{l}\text { Panicle } \\
\text { initiation } \\
\text { stage }\end{array}$ & $\begin{array}{c}\text { Harvest } \\
\text { stage }\end{array}$ & $\begin{array}{c}\text { Panicle } \\
\text { initiation } \\
\text { stage }\end{array}$ & $\begin{array}{c}\text { Harvest } \\
\text { stage }\end{array}$ \\
\hline $\mathrm{T}_{1}:$ Rec. NPK + FYM (No Zn and B application) & 5.87 & 5.83 & 2.40 & 2.29 & 16.81 & 16.04 \\
\hline $\mathrm{T}_{2}: \mathrm{RDF}+\mathrm{ZnSO}_{4} @ 10 \mathrm{~kg} \mathrm{ha}^{-1}$ & 5.90 & 5.85 & 2.54 & 2.36 & 20.41 & 19.92 \\
\hline $\mathrm{T}_{3}: \mathrm{RDF}+\mathrm{ZnSO}_{4} @ 20 \mathrm{~kg} \mathrm{ha}^{-1}$ & 5.98 & 5.91 & 2.57 & 2.41 & 21.21 & 20.33 \\
\hline $\mathrm{T}_{4}: \mathrm{RDF}+\mathrm{ZnSO}_{4} @ 30 \mathrm{~kg} \mathrm{ha}^{-1}$ & 5.95 & 5.88 & 2.52 & 2.34 & 22.82 & 21.27 \\
\hline $\mathrm{T}_{5}: \mathrm{RDF}+$ Borax @ $2 \mathrm{~kg} \mathrm{ha}^{-1}$ & 6.01 & 5.93 & 2.44 & 2.30 & 17.32 & 17.05 \\
\hline $\mathrm{T}_{6}: \mathrm{RDF}+$ Borax @ $4 \mathrm{~kg} \mathrm{ha}^{-1}$ & 6.06 & 5.98 & 2.48 & 2.33 & 18.19 & 17.79 \\
\hline $\mathrm{T}_{7}: \mathrm{RDF}+$ Borax @ $6 \mathrm{~kg} \mathrm{ha}^{-1}$ & 6.03 & 5.95 & 2.50 & 2.37 & 19.10 & 18.73 \\
\hline $\begin{array}{l}\text { T8: RDF + } \mathrm{ZnSO}_{4} @ 10 \mathrm{~kg} \mathrm{ha}^{-1}+\text { Borax @ } 2 \mathrm{~kg} \\
\mathrm{ha}^{-1}\end{array}$ & 6.04 & 5.96 & 2.53 & 2.35 & 20.65 & 19.51 \\
\hline $\begin{array}{l}\mathrm{T}_{9}: \mathrm{RDF}+\mathrm{ZnSO}_{4} @ 10 \mathrm{~kg} \mathrm{ha}^{-1}+\text { Borax @ } 4 \mathrm{~kg} \\
\mathrm{ha}^{-1}\end{array}$ & 6.07 & 5.97 & 2.56 & 2.38 & 22.07 & 21.11 \\
\hline $\begin{array}{l}\mathrm{T}_{10}: \mathrm{RDF}+\mathrm{ZnSO}_{4} @ 10 \mathrm{~kg} \mathrm{ha}^{-1}+\text { Borax @6 kg } \\
\mathrm{ha}^{-1}\end{array}$ & 6.09 & 6.00 & 2.59 & 2.41 & 22.60 & 21.77 \\
\hline $\begin{array}{l}\mathrm{T}_{11}: \mathrm{RDF}+\mathrm{ZnSO}_{4} @ 20 \mathrm{~kg} \mathrm{ha}^{-1}+\text { Borax @ } 2 \mathrm{~kg} \\
\mathrm{ha}^{-1}\end{array}$ & 6.12 & 6.03 & 2.61 & 2.40 & 22.26 & 21.20 \\
\hline $\begin{array}{l}\mathrm{T}_{12}: \mathrm{RDF}+\mathrm{ZnSO}_{4} @ 20 \mathrm{~kg} \mathrm{ha}^{-1}+\text { Borax @ } 4 \mathrm{~kg} \\
\mathrm{ha}^{-1}\end{array}$ & 6.15 & 6.07 & 2.64 & 2.42 & 23.01 & 22.42 \\
\hline $\begin{array}{l}\mathrm{T}_{13}: \mathrm{RDF}+\mathrm{ZnSO}_{4} @ 20 \mathrm{~kg} \mathrm{ha}^{-1}+\text { Borax @6 kg } \\
\mathrm{ha}^{-1}\end{array}$ & 6.18 & 6.10 & 2.66 & 2.44 & 22.88 & 21.87 \\
\hline $\begin{array}{l}\mathrm{T}_{14}: \mathrm{RDF}+\mathrm{ZnSO}_{4} @ 30 \mathrm{~kg} \mathrm{ha}{ }^{-1}+\text { Borax @ } 2 \mathrm{~kg} \\
\mathrm{ha}^{-1}\end{array}$ & 6.08 & 5.99 & 2.49 & 2.41 & 22.51 & 21.35 \\
\hline $\begin{array}{l}\mathrm{T}_{15}: \mathrm{RDF}+\mathrm{ZnSO}_{4} @ 30 \mathrm{~kg} \mathrm{ha}^{-1}+\text { Borax @ } 4 \mathrm{~kg} \\
\mathrm{ha}^{-1}\end{array}$ & 6.10 & 6.02 & 2.47 & 2.39 & 23.03 & 22.50 \\
\hline $\begin{array}{l}\mathrm{T}_{16}: \mathrm{RDF}+\mathrm{ZnSO}_{4} @ 30 \mathrm{~kg} \mathrm{ha}^{-1}+\text { Borax @6 kg } \\
\mathrm{ha}^{-1}\end{array}$ & 6.13 & 6.05 & 2.41 & 2.37 & 23.69 & 22.73 \\
\hline S.Em \pm & 0.02 & 0.02 & 0.04 & 0.04 & 0.39 & 0.47 \\
\hline C.D. at $5 \%$ & 0.06 & 0.05 & 0.11 & NS & 1.18 & 1.42 \\
\hline
\end{tabular}


Table.6 Effect of graded levels of zinc and boron on micronutrient status $\left(\mathrm{mg} \mathrm{kg}^{-1}\right)$ of soil at different growth stages of paddy at Naganahalli, Mysore district, Karnataka

\begin{tabular}{|c|c|c|c|c|c|c|c|c|c|c|}
\hline \multirow{2}{*}{ Treatments } & $\mathrm{Zn}$ & $\mathrm{Cu}$ & $\mathrm{Fe}$ & $\mathrm{Mn}$ & $\mathrm{B}$ & $\mathrm{Zn}$ & $\mathrm{Cu}$ & $\mathrm{Fe}$ & $\mathrm{Mn}$ & $\mathrm{B}$ \\
\hline & \multicolumn{5}{|c|}{ Panicle initiation stage } & \multicolumn{5}{|c|}{ Harvest stage } \\
\hline $\begin{array}{l}\mathrm{T}_{1}: \text { Rec. NPK + FYM (No Zn and B } \\
\text { application) }\end{array}$ & 1.31 & 1.38 & 4.67 & 2.16 & 0.63 & 1.21 & 1.31 & 4.62 & 2.12 & 0.60 \\
\hline $\mathrm{T}_{2}: \mathrm{RDF}+\mathrm{ZnSO}_{4} @ 10 \mathrm{~kg} \mathrm{ha}^{-1}$ & 2.84 & 1.33 & 4.37 & 2.13 & 0.66 & 2.73 & 1.25 & 4.22 & 2.08 & 0.63 \\
\hline $\mathrm{T}_{3}: \mathrm{RDF}+\mathrm{ZnSO}_{4} @ 20 \mathrm{~kg} \mathrm{ha}^{-1}$ & 3.03 & 1.28 & 4.21 & 2.10 & 0.72 & 2.94 & 1.21 & 4.13 & 2.05 & 0.68 \\
\hline $\mathrm{T}_{4}: \mathrm{RDF}+\mathrm{ZnSO}_{4} @ 30 \mathrm{~kg} \mathrm{ha}^{-1}$ & 2.95 & 1.24 & 4.11 & 2.08 & 0.67 & 2.81 & 1.19 & 4.02 & 2.05 & 0.65 \\
\hline $\mathrm{T}_{5}: \mathrm{RDF}+$ Borax @ $2 \mathrm{~kg} \mathrm{ha}^{-1}$ & 2.23 & 1.66 & 4.27 & 2.10 & 0.75 & 2.10 & 1.55 & 4.21 & 2.03 & 0.72 \\
\hline $\mathrm{T}_{6}: \mathrm{RDF}+$ Borax @ $4 \mathrm{~kg} \mathrm{ha}^{-1}$ & 2.70 & 1.77 & 4.12 & 2.07 & 0.82 & 2.57 & 1.70 & 4.08 & 2.01 & 0.77 \\
\hline $\mathrm{T}_{7}: \mathrm{RDF}+$ Borax @ $6 \mathrm{~kg} \mathrm{ha}^{-1}$ & 2.40 & 1.84 & 4.01 & 2.04 & 0.88 & 2.29 & 1.77 & 3.96 & 1.97 & 0.83 \\
\hline $\begin{array}{l}\mathrm{T}_{8}: \mathrm{RDF}+\mathrm{ZnSO}_{4} @ 10 \mathrm{~kg} \mathrm{ha}^{-1}+\text { Borax @ } 2 \\
\mathrm{~kg} \mathrm{ha}^{-1}\end{array}$ & 2.98 & 2.10 & 4.18 & 2.08 & 0.78 & 2.79 & 1.96 & 4.13 & 2.05 & 0.74 \\
\hline $\begin{array}{l}\text { T9: RDF + } \mathrm{ZnSO}_{4} @ 10 \mathrm{~kg} \mathrm{ha}^{-1}+\text { Borax @ } 4 \\
\mathrm{~kg} \mathrm{ha}^{-1}\end{array}$ & 3.07 & 2.24 & 4.04 & 2.06 & 0.85 & 2.91 & 2.19 & 3.89 & 2.00 & 0.80 \\
\hline $\begin{array}{l}\mathrm{T}_{10}: \mathrm{RDF}+\mathrm{ZnSO}_{4} @ 10 \mathrm{~kg} \mathrm{ha}^{-1}+\text { Borax @6 } \\
\mathrm{kg} \mathrm{ha}^{-1}\end{array}$ & 3.12 & 2.18 & 3.81 & 2.06 & 0.90 & 3.01 & 2.10 & 3.78 & 2.01 & 0.84 \\
\hline $\begin{array}{l}\mathrm{T}_{11}: \mathrm{RDF}+\mathrm{ZnSO}_{4} @ 20 \mathrm{~kg} \mathrm{ha}^{-1}+\text { Borax @ } 2 \\
\mathrm{~kg} \mathrm{ha}^{-1}\end{array}$ & 3.50 & 2.12 & 3.85 & 2.05 & 0.92 & 3.30 & 2.04 & 3.63 & 2.00 & 0.82 \\
\hline $\begin{array}{l}\mathrm{T}_{12}: \mathrm{RDF}+\mathrm{ZnSO}_{4} @ 20 \mathrm{~kg} \mathrm{ha}^{-1}+\text { Borax @ } 4 \\
\mathrm{~kg} \mathrm{ha}^{-1}\end{array}$ & 3.88 & 2.05 & 3.67 & 2.03 & 0.97 & 3.62 & 1.96 & 3.56 & 1.98 & 0.90 \\
\hline $\begin{array}{l}\mathrm{T}_{13}: \mathrm{RDF}+\mathrm{ZnSO}_{4} @ 20 \mathrm{~kg} \mathrm{ha}^{-1}+\text { Borax @6 } \\
\mathrm{kg} \mathrm{ha}^{-1}\end{array}$ & 3.69 & 1.93 & 3.36 & 2.00 & 0.94 & 3.45 & 1.81 & 3.29 & 1.98 & 0.88 \\
\hline $\begin{array}{l}\mathrm{T}_{14}: \mathrm{RDF}+\mathrm{ZnSO}_{4} @ 30 \mathrm{~kg} \mathrm{ha}^{-1}+\text { Borax @ } 2 \\
\mathrm{~kg} \mathrm{ha}^{-1}\end{array}$ & 3.61 & 2.03 & 3.31 & 2.01 & 0.90 & 3.35 & 1.94 & 3.25 & 1.96 & 0.84 \\
\hline $\begin{array}{l}\mathrm{T}_{15}: \mathrm{RDF}+\mathrm{ZnSO}_{4} @ 30 \mathrm{~kg} \mathrm{ha}^{-1}+\text { Borax @ } 4 \\
\mathrm{~kg} \mathrm{ha}^{-1}\end{array}$ & 3.47 & 1.94 & 3.30 & 1.99 & 0.87 & 3.30 & 1.86 & 3.25 & 1.93 & 0.82 \\
\hline $\begin{array}{l}\mathrm{T}_{16}: \mathrm{RDF}+\mathrm{ZnSO}_{4} @ 30 \mathrm{~kg} \mathrm{ha}^{-1}+\text { Borax @6 } \\
\mathrm{kg} \mathrm{ha}^{-1}\end{array}$ & 3.29 & 1.83 & 3.27 & 1.97 & 0.78 & 3.16 & 1.71 & 3.24 & 1.90 & 0.72 \\
\hline S.Em \pm & 0.15 & 0.04 & 0.09 & 0.01 & 0.02 & 0.12 & 0.05 & 0.20 & 0.02 & 0.02 \\
\hline C.D. at $5 \%$ & 0.45 & 0.11 & 0.28 & 0.04 & 0.05 & 0.35 & 0.15 & 0.61 & 0.05 & 0.06 \\
\hline
\end{tabular}


Status of calcium and magnesium was significantly influenced by the application of graded levels of zinc and boron at both the stages. Further significantly higher exchangeable $\mathrm{Ca}$ and $\mathrm{Mg}$ were noticed in $\mathrm{T}_{13}$ treatment $\left(\mathrm{RDF}+\mathrm{ZnSO}_{4} @ 20 \mathrm{~kg} \mathrm{ha}^{-1}+\right.$ Borax@6 kg ha ${ }^{-1}$ ) followed by $\mathrm{T}_{12}$ treatment at both stages but at harvest $\mathrm{Mg}$ showed nonsignificance. Lower exchangeable $\mathrm{Ca}$ and $\mathrm{Mg}$ was recorded in $\mathrm{T}_{1}$ treatment $(\mathrm{RDF}+\mathrm{FYM})$ at both stages. The combination of these nutrients could form synergistic relationship contributed for higher availability of these nutrients in soil. The exchangeable calcium and magnesium content of soil was higher at initial stage of the crop and declined at later stage. This may be attributed to uptake of calcium and magnesium by the growing plants as reported by Prakash et al., (1994).

The available $\mathrm{S}$ was influenced significantly by treatment effect in both stages. The plots treated with $\mathrm{ZnSO}_{4}$ had significantly higher content of available $S$ than those which were not treated with $\mathrm{ZnSO}_{4}$. The reason for this could be attributed to the release of $\mathrm{S}$ from $\mathrm{ZnSO}_{4}$ to the soil. After utilization of part of the released S from $\mathrm{ZnSO}_{4}$, the leftover of it might have contributed to the soil available pool. Since the levels of applied B were slightly low, but the B had significant effect on soil available sulphur.

\section{Micronutrient status of soil at panicle initiation and at harvest stages of paddy}

The status of micronutrient in soil as influenced by graded levels of zinc and boron in paddy are presented in Table 6 . In respect of available $\mathrm{Zn}$ and hot water soluble $\mathrm{B}$, the $\mathrm{T}_{12}\left(\mathrm{RDF}+\mathrm{ZnSO}_{4} @ 20 \mathrm{~kg} \mathrm{ha}^{-1}+\right.$ Borax @ 4 $\mathrm{kg} \mathrm{ha}{ }^{-1}$ ) treatment had significantly higher available $\mathrm{Zn}$ and $\mathrm{B}$ than $\mathrm{T}_{1}$ treatment (RDF+FYM) and followed by $\mathrm{T}_{13}$ treatment $\left(\mathrm{RDF}+\mathrm{ZnSO}_{4} @ 20 \mathrm{~kg} \mathrm{ha}^{-1}+\right.$ Borax @6 kg $\left.\mathrm{ha}^{-1}\right)$. However, the levels of $\mathrm{Zn}$ and $\mathrm{B}$ had significant effect on available $\mathrm{Zn}$ and $\mathrm{B}$ of soil at both stages. The difference in the quantities of applied $\mathrm{Zn}$ and $\mathrm{B}$ might be the reason for this and have good relation with synergistic in interaction.

Significantly higher $\mathrm{Cu}$ was recorded in $\mathrm{T}_{9}$ treatment $\left(\mathrm{RDF}+\mathrm{ZnSO}_{4} @ 10 \mathrm{~kg} \mathrm{ha}^{-1}+\right.$ Borax@4 kg ha ${ }^{-1}$ ) followed by $\mathrm{T}_{10}$ treatment $\left(\mathrm{RDF}+\mathrm{ZnSO}_{4} @ 10 \mathrm{~kg} \mathrm{ha}^{-1}+\right.$ Borax @6 kg $\mathrm{ha}^{-1}$ ). Lowest $\mathrm{Cu}$ in soil was recorded in $\mathrm{T}_{4}$ treatment $\left(\mathrm{RDF}+\mathrm{ZnSO}_{4} @ 30 \mathrm{~kg} \mathrm{ha}^{-1}\right)$ at both the stages. This might be due to antagonistic interaction of zinc and copper in soil (Tisdale et al., 1985).

The application of FYM revealed significant increase in $\mathrm{Fe}$ and $\mathrm{Mn}$ content in soil at both stages. The combined application of different levels of $\mathrm{Zn}$ and $\mathrm{B}$ treated plots showed low $\mathrm{Fe}$ and Mn content. Tisdale et al., (1985) reported that decreased availability of $\mathrm{Fe}$ and $\mathrm{Mn}$ in soils may be due to antagonistic interaction between zinc and manganese and boron and iron and manganese in soil.

It can be concluded that application of RDF + $\mathrm{ZnSO}_{4} @ 20 \mathrm{~kg} \mathrm{ha}{ }^{-1}+$ Borax @ $4 \mathrm{~kg} \mathrm{ha}^{-1}$ recorded significantly higher growth and yield of rice compared to other treatments. At both stages of crop, the significant increase in major, secondary and micronutrient status of soils was noticed among treatments due to application of RDF with graded levels of zinc and boron compared to $\mathrm{RDF}+\mathrm{FYM}$ treatments.

\section{References}

Abbas, M., Zahida, T. M., Uddin, R., Sajjid, I. and Akhlaq. 2013. Effect of zinc and boron fertilizers application on some physico-chemical attributes of five rice varieties grown in Agro-ecosystem of Sindh, Pakistan. American Eurasian J. Agril and environ. sci., 13(4): 433-439. 
Anonymous, 2015. Agricultural statistics at a glance. Directorate of Economics and Statistics, Ministry of Agriculture and Farmers Welfare, Government of India, New Delhi. Pp 84-85.

Ashour, N. I., and Reda, F. 1972. Effect of foliar application of some micro nutrients on growth and some physiological properties of sugar beet growth in winter season. Curr. Sci., 41(4): 146-147.

Bagewadi, P. C., Ramaiah, H., Krishnappa, N. and Balakrishna. 2003. Effect of nutrition and season on pod yield and seed quality of groundnut. Proceeding of National Workshop on Seed Technology of Groundnut at RARS, Raichur. 1: 77-88.

Balachandar, D., Nagarajan, P. and Gunasekaran. 2003. Effect of micronutrients on nodulation and yield of in acid soil condition. Legume Res., 26(2): 153-154.

Berger, K. C., and Truog, E. 1939. Boron determination in soils and plants. Ind. Eng. chem. Anal. Ed., 11: 540-545.

Chowdhury, A. R., Setty, T. K. P. and Nagarathna, T. K. 2010. Growth and yield of Sunflower (Helianthus annus L.) as influenced by micronutrient application in alfisols. Karnataka. $J$. Agri. Sci., 23(3): 495-496.

Christos Dordas. 2006. Foliar boron application affects lint and seed yield and improves seed quality of cotton grown on calcareous soils. Nutr. Cycl. Agroecosyst., 76: 19-28.

Das, K. P., 1992. Studies on the response of some micronutrients on growth and yield of groundnut. Proceeding of the Workshop on Micronutrients Seed Technolology. Parbhani.

Dashrath Singh, Mannikar, N. D. and Srinivas, N. G. 1976. Fertilizer value of indigenous rock phosphates compared with single super phosphate: laboratory incubation studies with farmyard manure. J. Indian Soc. Soil Sci., 24: 7880.

Fang, Y. L., Wang, Z., Xin, L., Zaho, X. and $\mathrm{Hu}, \mathrm{Q} .2008$. Effect of foliar application of zinc, selenium and iron fertilizers on nutrients concentration and yield of rice grain in china. J. Agri. Food Chem., 56: 2079-2084.

Hafeez, B., Khanif, Y. M., Samsuri, A. W., Radziah, O., Zakaria, W. and Saleem, M. 2013. Direct and Residual Effects of Zinc on Zinc-Efficient and ZincInefficient Rice Genotypes Grown under Low-Zinc-Content Submerged Acidic Conditions. Commun. Soil Sci. Plant Anal., 44: 2233-2252.

Jackson, M. L., 1973. Soil Chemical analysis. Prentice Hall of India (pvt.) Ltd., New Delhi.

Jyoti Sharma, Gupta A. K., Chandan Kumar and Gautam, R. K. S. 2013. Influence of zinc, calcium and boron on vegetative and flowering parameters of Gladiolus cv.Alberan. The Bioscan, 8(4): 11531158.

Lindsay, W. L., and Norwell, W. A. 1978. Development of DTPA soil test for zinc, iron, manganese and copper. Soil Sci. Soc. Am. J., 42: 421-428.

Muhammad Arif, Muhammad Asif Shehzad, Fiaz Bashir, Muhammad Tasneem, Ghulam Yasin and Munawar Iqbal. 2012. Boron, zinc and microtone effects on growth, chlorophyll contents and yield attributes in rice (Oryza sativa $L$ ) cultivar. African J. Biotech., 11(48): 10851-10858.

Page, A. L., Miller, R.H. and Kenay, D. R. 1982. Methods of soil analysis. Part-2 Soil Science of America, Inc. Publs. Madison, Wisconsin, USA.

Piper, C. S., 1966. Soil and Plant analysis. Hans Publishers. Bombay.

Prakash, T. R., Badrinath, M. S. and Mir Khamer Ali. 1994. Relative efficiency 
of different phosphorus sources as influenced by liming on the yield and uptake by rice on Oxisols. J. Indian Soc. Soil Sci., 42: 271-273.

Pratima Sinha, Radha Jain and Chitralekha Chatterjee. 2000. Interactive effect of boron and zinc on growth and metabolism of Mustard. Commun. Soil Sci. Plant Anal., 31(1\&2): 41-49.

Quddus, M. A., Rashid, M. H., Hassain, M. A. and Naser, H. M. 2011. Effect of zinc and boron on yield and yield contributing characters of mungbean in low Ganges river flood plain soil at Madaripur, Bangladesh. Bangladesh J. Agril. Res., 36(1): 75-85.

Raghuveer Rao, D., Subrhamanyam, B., Sailaja, R. P., Singh, V., Ravichandran and Sudershan Rao, G. V. 2013. Influence of boron on spikelet fertility under varied soil conditions in rice genotypes. J. Pl. Nutri., 36: 390-400.

Rao, C. P., and Shukla, D. N. 1997. Physiological parameters and yield of transplanted rice in combination with zinc. Indian J. Agric. Sci., 67(5): 215217.

Sharma, S.K., 1995. Response of boron and calcium nutrition on plant growth, fruit and seed of tomato. Vegetable Sci., 22:27-29.

Subbaiah, B. Y., and Asija, G. L. 1956. A rapid procedure for the estimation of available nitrogen in soils. Curr. Sci., 25: 259-260.

Subrahmaniyan, K., Kalaiselven, P. and Arulmozhi, N. 2001. Response of confectionary groundnut to micronutrients. Legume Res., 24(2):139140.

Tisdale, S. L., Nelson, W. L. and Beaton, J. D. 1985. In: Soil fertility and fertilizers. Published by Macmillan publishing company, New York.

Walkley, A. J., and Black, I. A. 1934. An examination of the degtjareff method for determining soil organic matter and a proposed modification of the chromic acid titration method. J. Soil Sci., 37: 29-38.

\section{How to cite this article:}

Mahendra Kumar, M.B., C.T. Subbarayappa and Ramamurthy, V. 2017. Effect of Graded Levels of Zinc and Boron on Growth, Yield and Chemical Properties of Soils under Paddy. Int.J.Curr.Microbiol.App.Sci. 6(10): 1185-1196. doi: https://doi.org/10.20546/ijcmas.2017.610.143 